\title{
Pharmacogenomics in the clinic: genetic polymorphism contributing to venlafaxine-associated heart failure
}

\author{
Harleen Singh*,1,3, Barent DuBois², Zain Al-Jammali ${ }^{1}$ iD \& Thomas Barrett ${ }^{3}$ \\ ${ }^{1}$ Department of Pharmacy Practice, College of Pharmacy, Oregon State University, Portland, OR 97201, USA \\ ${ }^{2}$ QPS LLC, Pencader Corporate Center, 110 Executive drive, Newark, DE 19702, USA \\ ${ }^{3}$ Department of Internal Medicine, The Veterans Affairs Portland Health Care System, Portland, OR 97239, USA \\ *Author for correspondence: Tel.: +1 503494 1483, Mobile: +1 614354 6863; singhh@ohsu.edu
}

First draft submitted: 13 June 2019; Accepted for publication: 6 September 2019; Published online: 4 December 2019

Keywords: antidepressants $\bullet$ CYP2D6 $\bullet$ CYPC 19 $\bullet$ CYP polymorphism $\bullet$ drug induced cardiomyopathy $\bullet$ heart failure - SNRI • Venlafaxine

Pharmacogenomic tests are now commercially available, but it is difficult to know which patients and drugs should trigger consideration for pharmacogenomic evaluation. Venlafaxine is a widely used serotonin-norepinephrine reuptake inhibitor indicated for the treatment of major depression disorder, anxiety and pain disorders [1]. Genetic variation is thought to play an important role in patient risk for cardiotoxicity by both acute and prolonged exposure to venlafaxine. Polymorphisms and drug-drug interactions with CYP2D6, and to some extent CYP2C19, have been shown to significantly influence the pharmacokinetics and metabolic clearance of venlafaxine and the formation of its active metabolite, O-desmethylvenlafaxine (ODV) [2-5]. Venlafaxine and ODV are both potent inhibitors of serotonin and norepinephrine reuptake. Other hepatic enzymes (CYP3A4, CYP2C19 and CYP2C9) metabolize venlafaxine and ODV to minor, less active metabolites (Figure 1).

Several case reports have shown an association between cardiomyopathy and a poor CYP2D6 metabolizer status in patients taking venlafaxine [6-8]. While cardiac events with venlafaxine are considered rare even at high doses, evidence suggests drug-induced cardiomyopathy and heart failure in instances of overdose and regular dosing of venlafaxine. Resolution of symptoms upon cessation of venlafaxine therapy further supports cardiovascular adverse events related to venlafaxine. The exact mechanism of cardiotoxicity remains unknown; however, some hypotheses have been made. One theory is the hypertrophic effects of dose-related sustained hypertension. Likewise, myocardial damage due to increased levels of catecholamine with inhibition of dopamine and norepinephrine reuptake is reported by the current literature [9]. The surge in catecholamine levels eventually leads to the development of cardiomyopathy resulting in left ventricular dysfunction. Patient genotyping allows for a fairly reliable estimation of CYP2D6 and CYP2C19 phenotypic status with respect to venlafaxine and ODV exposure, especially among 'poor' and 'extensive (normal)' metabolizers [4,5]. However, there are few case reports speculating on the clinical significance for an 'intermediate' CYP2D6 metabolizer; Vinetti et al. report an intermediate CYP2D6 metabolizer who presented with severe acute cardiomyopathy following a venlafaxine overdose [8]. We report a patient who developed nonischemic cardiomyopathy after 4 years of venlafaxine treatment for depression and anxiety, and who is a $C Y P 2 D 6$ intermediate metabolizer and CYP2C19 extensive metabolizer.

\section{Case report}

A 37-year-old male presented to the emergency department (ED) in 2013 with nausea, vomiting, abdominal distention, dyspnea on exertion and a 15 -pound weight gain 3 weeks prior to admission despite poor oral intake. The patient was seen in the ED 6 days prior and received a 5-day course of azithromycin for suspected pneumonia. Vitals on admission were: temperature of $97^{\circ} \mathrm{F}, 98 \%$ RA, weight $290 \mathrm{lbs}, 72$ inches height, BMI $39.3 \mathrm{~kg} / \mathrm{m}^{2}$, blood pressure $174 / 131 \mathrm{mmHg}$ and heart rate 117 beats/minute. Physical exam findings included jugular venous pressure of $18 \mathrm{~cm}$ above the right atrium and decreased breath sounds at half of the right thorax. Serum creatinine $1.4 \mathrm{mg} / \mathrm{dl}$ (previously recorded at $0.9 \mathrm{mg} / \mathrm{dl}$ ), NT-proBNP $4921 \mathrm{pg} / \mathrm{ml}$. The diagnosis was acute decompensated heart failure (HF).

Future 8 Medicine 


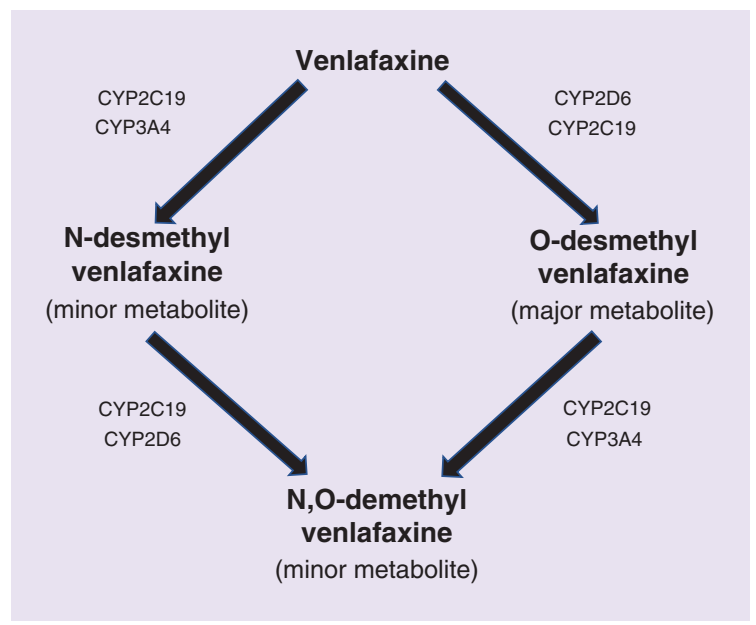

Figure 1. Velafaxine metabolism: influence of CYP2D6 and CYP2C19 isoenzymes.

\section{Table 1. CYP 2D6 and 2C19 allele activity status.}

\begin{tabular}{|c|c|c|c|c|}
\hline & Normal activity & Decreased activity & Increased activity & Null activity \\
\hline CYP 2D6 & $\begin{array}{l}\dagger 1 \\
\dagger 2 \\
\dagger 2 \mathrm{~A} \\
\dagger 33 \\
\dagger 35\end{array}$ & $\begin{array}{l}\dagger 9 \\
\dagger 10 \\
\dagger 14 \\
\dagger 17 \\
\dagger 17 \\
\dagger 29 \\
\dagger 41\end{array}$ & $\mathrm{~N} / \mathrm{A}$ & $\begin{array}{l}\dagger 3-{ }^{\dagger} 8 \\
\dagger 11-\dagger 13 \\
\dagger 15 \\
\dagger 19-{ }^{\dagger} 21 \\
\dagger 38 \\
\dagger 40 \\
\dagger 42\end{array}$ \\
\hline CYP 2C19§ & $\begin{array}{l}\dagger 1 \\
\dagger 11 \\
\dagger 13 \\
\dagger 15 \\
\dagger 18 \\
\dagger 28\end{array}$ & $\begin{array}{l}\dagger 9 \\
\dagger 10 \\
\dagger 16 \\
\dagger 19 \\
\dagger 25 \\
\dagger 26\end{array}$ & $\dagger 17$ & $\begin{array}{l}\dagger 2-\dagger 8 \\
\dagger 22 \\
\dagger 24 \\
\dagger 35\end{array}$ \\
\hline $\begin{array}{l}\text { †Indicates all } \\
\text { ‡Pharmvar. } \\
\text { \$Pharmvar. }\end{array}$ & $\begin{array}{l}\text { er 2019). } \\
\text { ber 2019). }\end{array}$ & & & \\
\hline
\end{tabular}

Social history included chronic alcohol use (4-6 cans of beer/night) that was stopped 3 weeks prior to admission due to nausea and vomiting. Patient had not been diagnosed with hypertension in the past but had hypertensive urgency at admission. Outpatient medications included buspirone $15 \mathrm{mg}$ twice daily and venlafaxine $150 \mathrm{mg}$ twice daily for 4 years. Chest computed tomography showed pleural effusion in the right lung, right lower lobe consolidation with air bronchograms and potential cardiomegaly; thoracentesis categorized the effusion as transudative. Transthoracic echocardiogram showed left ventricular ejection fraction of $25 \%$ with signs of mild/moderate mitral regurgitation and severe tricuspid regurgitation. The etiology of HF was presumed to be nonischemic dilated cardiomyopathy. The patient was started on intravenous furosemide, metoprolol tartrate, hydralazine, isosorbide dinitrate and digoxin. While hospitalized, the patient diuresed 20 pounds. Cardiac catheterization showed normal coronary arteries, severely elevated right atrial pressure $(22 \mathrm{mmHg})$, elevated pulmonary capillary wedge pressure $(23 \mathrm{mmHg})$, reduced cardiac index $\left(1.5 \mathrm{l} / \mathrm{min} / \mathrm{m}^{2}\right)$, systemic vascular resistance $\left(1337 \mathrm{dyn}-\mathrm{s} / \mathrm{cm}^{5}\right)$ and pulmonary vascular resistance $\left(1.1\right.$ dyn-s/ $\left.\mathrm{cm}^{5}\right)$.

The patient was discharged on day 12, with a 2-week HF clinic follow-up. During the clinic visit, consideration was given to the possibility of being a poor CYP2D 6 metabolizer resulting in high levels of venlafaxine and therefore a high catecholamine level causing HF. Genotyping showed that the patient was CYP2D6 (CYP2D6 * $\left.1^{*} 4\right)$ intermediate metabolizer and CYP2C19 (CYP2C19 $\left.{ }^{*} 1^{*} 17\right)$ rapid metabolizer (See Table 1). Polymerase chain reaction with allele-specific primers that specifically determined $C Y P 2 D 6{ }^{*} 2 A, 1 C,{ }^{*} 12,{ }^{*} 15,{ }^{*} 11,{ }^{*} 17,{ }^{*} 6,{ }^{*} 8 \backslash{ }^{*} 14,{ }^{*} 4$, ${ }^{*} 3,{ }^{*} 9,{ }^{*} 2,{ }^{*} 7, * 41$ was used. CYP2D 6 gene deletion and gene duplication were performed following polymerase chain reaction amplification. CYP2C19 testing indicated that the patient carries one copy of the CYP2C19*17 variant and none of the tested deficiency alleles in the CYP2C19 gene. The testing assay can detect the wild-type allele $\left(C Y P 2 C 19^{*} 1\right)$ as well as ten common mutations in the CYP2C19 gene: CYP2C19*2, CYP2C19*3, CYP2C19*4, 
CYP2C19*5, CYP2C19*6, CYP2C19*7, CYP2C19*8, CYP2C19*9, CYP2C19*12 and CYP2C19*17. Patient's plasma norepinephrine $(694 \mathrm{pg} / \mathrm{ml})$, reference $<420)$ levels were high. Venlafaxine was tapered down and discontinued over the following month. Repeat CT chest showed complete resolution of right lower lobe infiltrate and effusion at 1 month, and repeat echocardiogram at 3 months showed resolution of systolic dysfunction with left ventricular ejection fraction of 55-60\% without further episodes of fluid accumulation.

\section{Discussion}

We report a case of cardiomyopathy we suspect was due to venlafaxine toxicity. Before this incident, our patient had no previous symptoms associated with heart failure. His nausea and emesis were thought to be from gut edema from fluid accumulation, as was his dyspnea on exertion and increased abdominal girth. His symptoms at presentation were potentially consistent with drug-induced pneumonitis and heart failure, which have been associated with venlafaxine toxicity. Another possible explanation for the etiology of his nonischemic heart failure is alcoholic cardiomyopathy given that his social history included chronic alcohol abuse. If alcohol were the only culprit, it seems unlikely his echocardiogram would have shown resolution of cardiac dysfunction after a 2-month follow-up. During this time, the patient was tapered off venlafaxine therapy when genotyping determined our patient to be a CYP2D6 intermediate metabolizer $\left(C Y P 2 D 6^{*} 1 /^{*} 4\right)$. The cessation of venlafaxine therapy, and the combined potential for impaired metabolism - CYP2D6 genotype is strongly suggestive of venlafaxine cardiomyopathy.

Two enzymes, CYP2D6 and CYP2C19, contribute significantly to the metabolism of venlafaxine [3,8,10]. Genetic polymorphisms in these enzymes can result in a loss or a gain of function. There are more reports suggesting a 'poor' CYP2D6 genotyping, meaning two null alleles, is at particular risk for venlafaxine toxicity. In this case, we describe a patient who is an intermediate CYP2D6 metabolizer (one null copy and one normal copy), and rapid CYP2C19 metabolizer (one normal copy and one enhanced gain of function copy). It is generally assumed such individuals may be at greater risk for drug interactions given that lower enzyme expression is more saturable. The metabolic clearance of venlafaxine should be studied within the context of both CYP2D6 and CYP2C19 activity as it has been shown that both are involved in venlafaxine biotransformation, and a case report of fatal venlafaxine poisoning was observed in a young man with a both 'poor' CYP2D6 and CYP2C19 genotypes [4,7]. We identified two reports of patients with one null CYP2D6 allele who presented with adverse reactions with venlafaxine therapy $[6,8]$. The report by Chau et al. involved a patient with two novel variants of CYP2D6 and CYP2C19 (but one functional copy of each), implying an impaired metabolic phenotype [6]. Vinetti et al. report a patient with a $C Y P 2 D 6^{*} 1 /{ }^{*}$, very similar to our own and also an 'intermediate' CYP2D6 metabolizer [8]. Most interestingly, our patient and the case in Vinetti et al. had a CYP2C19* 17 allele, which is associated with increased activity and a 'rapid' CYP2C19 genotype.

These data are suggestive that CYP2C19 may not completely compensate diminished CYP2D6 function, or may play a role in producing a metabolite which is additively or synergistically cardiotoxic with its parent. The clinical significance of the intermediate CYP2D6 genotype, and to what extent enhanced CYP2C19 activity can compensate is not well understood. One reason it may have taken this patient years to develop heart failure is that the CYP2D6 was intermediate not poor metabolizer. While these patients may tolerate venlafaxine therapy for months, even years, caution should be exercised as case reports indicate risk for heart failure and death even with normal dosages [11]. Given the strong association with venlafaxine-induced heart failure, an alternative therapy should be considered. The Dutch Pharmacogenetics Working Group recommends that for poor and intermediate venlafaxine metabolizers of CYP2D6, an alternative drug should be used (e.g., citalopram, sertraline), or the dose of venlafaxine should be adjusted based on clinical response, and ODV plasma levels should be monitored [12]. On the contrary, the US FDA recommends no dose adjustments for poor and extensive (normal) metabolizers because the total exposure (AUC) of venlafaxine and ODV is similar in both groups [13].

This case highlights the need for more detailed studies on the clinical significance of CYP2C19 polymorphisms and their interaction with CYP2D6. Clinicians should be aware that the 'intermediate' CYP2D6 genotype may have clinical relevancy for venlafaxine cardiomyopathy, particularly in patients on concomitant medications which are substrates for CYP2D6, or who abuse alcohol. Our main recommendation is that if a clinician is seeing a younger patient with a nonischemic cardiomyopathy also taking venlafaxine, then consideration for pharmacogenomic testing for CYP2D6 and CYP2C19 should be given. Furthermore, pharmacogenetics-based prescribing guidelines recommend to consider testing of CYP2D6 prior to initiating venlafaxine [12]. 


\section{Author contributions}

All authors vouched to be accountable for all aspects of the work in ensuring that questions related to the accuracy or integrity of any part of the work are appropriately investigated and resolved. H Singh and B Dubois contributed to the acquisition, analysis or interpretation of data for the work as well as the manuscript drafting. T Barret provided substantial contributions to conception or design of the work. Z Al-Jammali, B Dubois and T Barrett contributed to the final review and approval of the manuscript version to be published.

\section{Financial \& competing interests disclosure}

The authors have no relevant affiliations or financial involvement with any organization or entity with a financial interest in or financial conflict with the subject matter or materials discussed in the manuscript. This includes employment, consultancies, honoraria, stock ownership or options, expert testimony, grants or patents received or pending, or royalties.

No writing assistance was utilized in the production of this manuscript.

\section{References}

1. Bandelow B, Sher L, Bunevicius R et al. Guidelines for the pharmacological treatment of anxiety disorders, obsessive-compulsive disorder and posttraumatic stress disorder in primary care. Int. J. Psychiatry Clin. Pract. 16(2), 77-84 (2012).

2. Kandasamy M, Srinivas P, Subramaniam K et al. Differential outcomes from metabolic ratios in the identification of CYP2D6 phenotypes - focus on venlafaxine and O-desmethylvenlafaxine. Eur. J. Clin. Pharmacol. 66(9), 879-887 (2010).

3. Lessard E, Yessine MA, Hamelin BA, O'Hara G, Leblanc J, Turgeon J. Influence of CYP2D6 activity on the disposition and cardiovascular toxicity of the antidepressant agent venlafaxine in humans. Pharmacogenetics 9(4), 435-443 (1999).

4. Mcalpine DE, Biernacka JM, Mrazek DA et al. Effect of cytochrome P450 enzyme polymorphisms on pharmacokinetics of venlafaxine. Ther. Drug Monit. 33(1), 14-20 (2011).

5. Shams ME, Arneth B, Hiemke C et al. CYP2D6 polymorphism and clinical effect of the antidepressant venlafaxine. J. Clin. Pharm. Ther. 31(5), 493-502 (2006).

6. Chua EW, Foulds J, Miller AL, Kennedy MA. Novel CYP2D6 and CYP2C19 variants identified in a patient with adverse reactions towards venlafaxine monotherapy and dual therapy with nortriptyline and fluoxetine. Pharmacogenet. Genomics 23(9), 494-497 (2013)

7. Jornil J, Nielsen TS, Rosendal I et al. A poor metabolizer of both CYP2C19 and CYP2D6 identified by mechanistic pharmacokinetic simulation in a fatal drug poisoning case involving venlafaxine. Forensic Sci. Int. 226(1-3), e26-31 (2013).

8. Vinetti M, Haufroid V, Capron A, Classen JF, Marchandise S, Hantson P. Severe acute cardiomyopathy associated with venlafaxine overdose and possible role of CYP2D6 and CYP2C19 polymorphisms. Clin. Toxicol. 49(9), 865-869 (2011).

9. Batista M, Dugernier T, Simon M et al. The spectrum of acute heart failure after venlafaxine overdose. Clin. Toxicol. 51(2), 92-95 (2013).

10. Karlsson L, Zackrisson AL, Josefsson M, Carlsson B, Green H, Kugelberg FC. Influence of CYP2D6 and CYP2C19 genotypes on venlafaxine metabolic ratios and stereoselective metabolism in forensic autopsy cases. Pharmacogenomics J. 15(2), 165-171 (2015).

11. Drent M, Singh S, Gorgels AP et al. Drug-induced pneumonitis and heart failure simultaneously associated with venlafaxine. Am. J. Respir. Crit. Care Med. 167(7), 958-961 (2003).

12. Pharmgkb. Annotation of DPWG Guideline for venlafaxine and CYP2D6 (2018). www.pharmgkb.org/chemical/PA451866/guidelineAnnotation/PA166104968

13. Lexicomp. Venlafaxine. Wolters Kluwer Clinical Drug Information, Inc., OH, USA (2019). http://online.lexi.com/lco/action/doc/retri eve/docid/patch_f/7862?searchUrl=\%2Flco\%2Faction\%2Fsearch\%3Fq\%3DVenlafaxine\%26t\%3Dname\%26va\%3Dvenlafax 